\title{
Artocarpus: Um Gênero Exótico de Grande Bioatividade
}

\author{
Valéria de Jesus Pereira ${ }^{1}$, Maria Auxiliadora Coelho Kaplan ${ }^{1}$ \\ ${ }^{1}$ Núcleo de Pesquisas de Produtos Naturais, Universidade Federal do Rio de Janeiro - UFRJ, Rio de Janeiro/RJ, Brasil
}

\begin{abstract}
RESUMO
O gênero Artocarpus está representado por 50 espécies, que estão distribuídas, principalmente, nas regiões tropicais asiáticas, sendo utilizado pela medicina popular, nessa área endêmica, como tratamento ou prevenção de inflamações, malária e vitiligo. As investigações fitoquímicas e biológicas em 34 espécies resultaram no isolamento de 369 substâncias, representadas, principalmente, por flavonoides, sendo flavona o tipo mais característico.
\end{abstract}

Palavras-chave: metabólitos especiais, Artocarpus, bioatividade.

\section{The High Bioactivity of Artocarpus - An Exotic Genus}

\begin{abstract}
Artocarpus genus is represented by 50 species distributed mainly in the tropical regions of Asia where they are used by folk medicine for the treatment or prevention of inflammations, malaria and vitiligo in these endemic areas. Phytochemical and biological investigations in 34 species resulted in the isolation of 369 compounds, mostly represented by flavonoid aglicones, with flavone being the most common.
\end{abstract}

Keywords: special metabolites, Artocarpus, bioactivity.

\section{INTRODUÇÃO}

A família Moraceae, uma valiosa Angiospermae, é constituída por 37 gêneros e cerca de 1.100 espécies, de ampla ocorrência nas regiões tropicais do planeta (Datwyler \& Weiblen, 2004); encontra-se, segundo os critérios de classificação da Angiosperm Philogeny Group-III (APG-III), dentro da ordem Rosales (Bremer et al., 2009). Artocarpus é um gênero de hábito arbóreo dessa família, contendo cerca de 50 espécies (Nomura et al., 1998). Plantas desse gênero são oriundas de regiões tropicais e subtropicais do continente asiático, e uma delas, cultivada no Brasil como exótica, aparece em listas oficiais como invasora de habitats florestais (Siqueira, 2006; Instituto Horus, 2012).
Artocarpus heterophyllus Lamk foi introduzida no Brasil pela Coroa Portuguesa no século XVII (Prestes, 2000), dentro de uma política mercantilista que visava à aclimatação de plantas e especiarias de origem asiática. Essa espécie, popularmente conhecida por jaqueira, adaptou-se facilmente ao território brasileiro por diversos fatores: grande produção anual de frutos, ausência de predadores específicos e efeito alelopático, bem como por servir de alimento para várias espécies de animais nativos. Entre outras espécies de plantas exóticas, foi utilizada pelo paisagista francês Auguste Glaziou (1833-1906), que a serviço do imperador Pedro II projetou vários jardins no País, dentre os quais o Campo de Santana 
e a Quinta da Boa Vista, ambos no Rio de Janeiro (Santos et al., 2008).

Do ponto de vista econômico, as espécies de Artocarpus são bastante conhecidas pelos seus frutos, como a jaca, cuja parte comestível corresponde, na verdade, às paredes desenvolvidas dos ovários, popularmente conhecidos por bagos. Há, particularmente, duas variedades de jaca, de acordo com a consistência de seus bagos: a mole e a dura, sendo a primeira mais doce e macia, conhecida pelo nome de jaca-manteiga (Peixoto \& Toledo, 2002). Outra fruta bastante apreciada é a fruta-pão, Artocarpus altilis, alimento típico das Regiões Norte e Nordeste do Brasil. As espécies Artocarpus hirsutus Lam. e Artocarpus champeden Spreng. destacam-se pelo valor econômico de suas madeiras e pelo seu uso na medicina popular dos países de origem, para o tratamento de malária, entre outras ocorrências patológicas.

\section{DIVERSIDADE METABÓLICA DE ESPÉCIES DE ARTOCARPUS}

\subsection{Flavonoides}

Dentre todos os metabólitos especiais, a classe dos flavonoides tem no gênero Artocarpus uma grande expressividade, pois compreende cerca de $75 \%$ das substâncias isoladas.

As flavonas (1-21) representam o maior grupo de metabólitos estudados, seja com estruturas simples com ou sem substituintes prenila livres, seja levando a piranoflavonas, furanoflavonas e oxepinoflavonas, entre outras variedades (Figura 1). Em comum entre as flavonas, há uma forte presença de substituintes isoprenila ou geranila em C3 e, eventualmente, em C6 e C8. Por outro lado, nota-se um padrão de substituição 2',4' dioxigenado ou 2',4',5' trioxigenado no anel B do esqueleto flavonoídico, encontrando-se, por vezes, a presença de grupos metoxila, isoprenila e geranila nas posições C2' e C4'. Algumas estruturas apresentam anéis que podem ter seis ou sete átomos de carbono resultantes da ligação do grupo isoprenila em C3 com C2'.

Em menor número, as flavanonas (22-24) aparecem também com inúmeros grupos substituintes dos tipos isoprenila e geranila em C6 e C8. Encontram-se algumas estruturas cujo grupo prenila em C5' faz gerar outra ciclização no anel B (Figura 2).

Chalconas (25-30) e Diidrochalconas (31-35) aparecem com farta ocorrência de grupos geranila e isoprenila nas posições C3', C5' e C2, formando anéis de cinco ou seis átomos com C4' ou C3 (Figura 3)

As estruturas de proantocianidinas (36), catequinas (37), auronas glicosiladas (38) e isoflavonas (39) encontram-se na Figura 4. É interessante observar que o padrão de oxigenação manteve-se em todas essas classes, verificando-se, no entanto, apenas na isoflavona a substituição por grupo prenila nas posições típicas.

\subsection{Outras substâncias fenólicas produzidas por plantas de espécies de Artocarpus}

Outras classes de substâncias fenólicas são descritas para representantes de Artocarpus, tais como: xantonas (40-43), 2-arilbenzofuranos (4452), estilbenos (53-56), alguns adutos de DielsAlder de diidroprenilchalcona com chalcona (5758), derivados de etilbenos prenilados, dímeros de estilbeno (59-62), derivados de Neolignanas (63), e várias outras substâncias (64-67), cujas estruturas aparecem representadas na Figura 5. Há nesse grupo uma grande variedade de quinonas, cromonas e a presença constante de grupos prenila e geranila.

\subsection{Terpenoides e Fitoesteroides}

O perfil químico traçado pelos trabalhos analisados apresentou especialmente esqueletos do tipo lupano (68), ursano (69), oleanano (70), cicloartano (71) e glutinano (72), dentro da classe dos triterpenos. Os sesquiterpenos apresentaram apenas dois tipos de esqueletos básicos, o germacrano (73) e o longifoleno (74), de ocorrência restrita a Artocarpus heterophyllus (Wang et al., 2007). De Artocarpus communis, A. altilis, A. champeden, A. heterophyllus e A. hypargyreus, foram registrados esqueletos colestano (75) e sua versão betaglicosilada foi descrita em Artocarpus sepicanus, A. altilis e A. hypargyreus. Somente a espécie Artocarpus heterophyllus apresentou esqueleto estigmastano (76) (Yao \& Min, 2005) (Figura 6). 

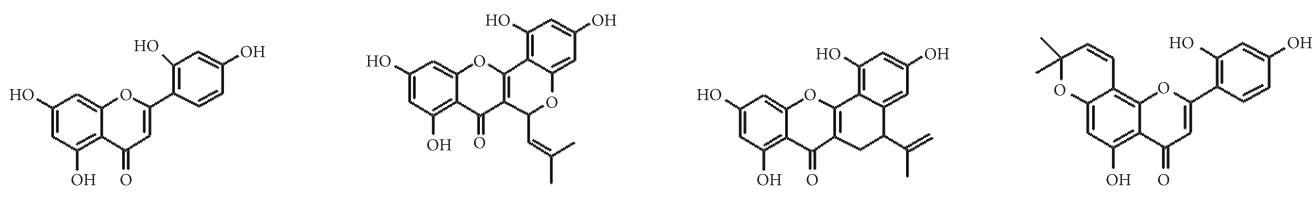

2

3

4

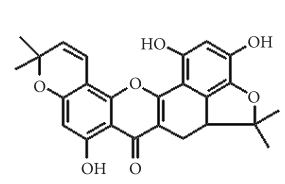

5

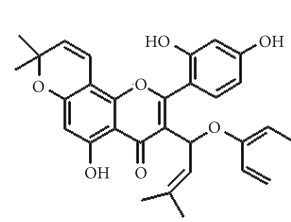

9<smiles>CC(C)=CCc1c2c(c(O)c3c(=O)c4c(oc13)-c1ccc(O)cc1OC(C(C)(C)C)C4)CC(C(C)(C)C)O2</smiles>

13<smiles>C=C(C)C1Cc2cc(-c3cc(=O)c4c(O)c5c(cc4o3)OC(C)(C)C=C5)cc(O)c2O1</smiles>

16<smiles>C=C(C)C1Cc2c(oc3cc4c(c(O)c3c2=O)C=CC(C)(C)O4)-c2c(O)cc(O)c(O)c21</smiles>

19

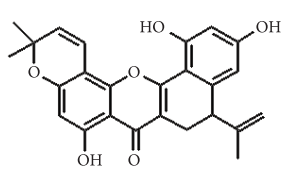

6

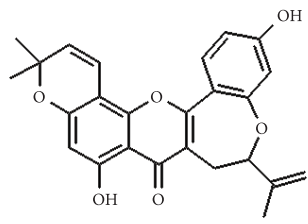

10

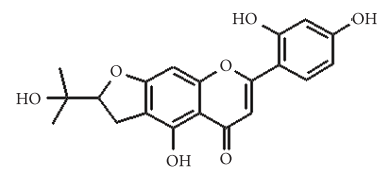<smiles>CC(C)=C1Cc2cc(-c3cc(=O)c4c(O)c5c(cc4o3)OC(C)(C)C=C5)cc(O)c2O1</smiles><smiles>CC1(C)C=Cc2c(O)cc(-c3cc(=O)c4c(O)c5c(c(O)c4o3)OC(C)(C)C=C5)c(O)c2O1</smiles>

14

17

20

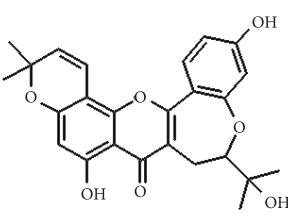

11

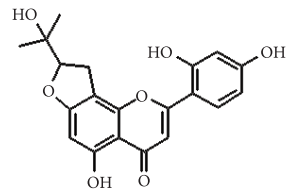

15

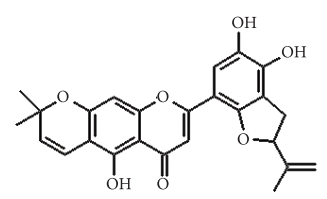

18

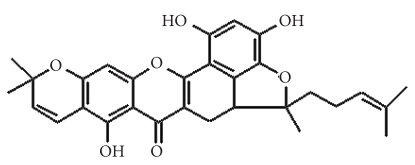

21

Figura 1. Estruturas típicas de flavonas de espécies de Artocarpus.

Figure 1. Artocarpus species typical flavon structures.<smiles>CC(C)=CCc1cc([C@H]2CC(=O)c3c(cc(O)c(CC=C(C)C)c3O)O2)ccc1O</smiles>

22<smiles>CC1(C)C=Cc2cc(C3CC(=O)c4c(O)cc(O)cc4O3)c(O)cc2O1</smiles>

23<smiles>CC1(C)C=Cc2c(cc(O)c3c2OC(c2ccc(O)cc2)CC3=O)O1</smiles>

24

Figura 2. Estruturas típicas de flavanonas de espécies de Artocarpus.

Figure 2. Artocarpus species typical flavanon structures. 
<smiles>C=C(C)C1Cc2c(ccc(C(=O)/C=C/c3ccc(O)c(O)c3)c2O)OC1C(=O)/C=C/c1ccc(O)c2c1C=CC(C)(CC=C(C)C)O2</smiles>

25<smiles>CC(C)=CCC[C@]1(C)C=Cc2cc(C(=O)/C=C/c3ccc(O)cc3)c(O)cc2O1</smiles>

29
26<smiles>CC(C)(O)C1Oc2ccc(/C=C/C(=O)c3ccc(O)cc3O)cc2[C@H]1O</smiles>

30
27<smiles>CC(C)=CCC1(C)Cc2c(CCC(=O)c3ccc(O)cc3O)ccc(O)c2OC1(C)C</smiles>

31<smiles>O=C(CCc1ccc(O)c(O)c1)c1ccc(O)cc1O</smiles>

32<smiles>CC(C)=CCC1(C)C=Cc2c(CCC(=O)c3ccc(O)cc3O)ccc(O)c2O1</smiles>

33<smiles>CC1(/C=C/C2OC2(C)C)C=Cc2c(CCC(=O)c3ccc(O)cc3O)ccc(O)c2O1</smiles>

34<smiles>CC1=CC2c3c(CCC(=O)c4ccc(O)cc4O)ccc(O)c3OC(C)(C)C2CC1</smiles>

35

Figura 3. Estruturas típicas de Chalconas e Diidrochalconas de espécies de Artocarpus.

Figure 3. Artocarpus species typical Chalcones and Dihydrochalcones structures.

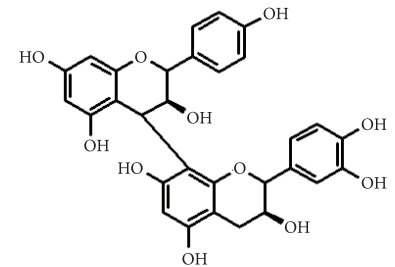

36<smiles>Oc1ccc(C2Oc3cc(O)cc(O)c3CC2O)cc1</smiles>

37

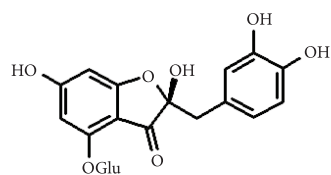

38

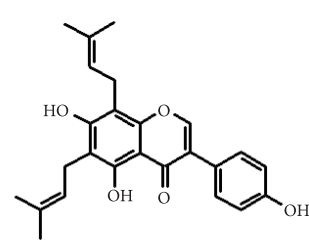

39

$\mathrm{R}=\mathrm{H}, \mathrm{R}=$ Ramnosila/Rhamnosil

Figura 4. Estruturas típícas de outros flavonoides de espécies de Artocarpus.

Figure 4. Other flavonoid typical structures of Artocarpus species.

\section{OCORRÊNCIA E ATIVIDADE BIOLÓGICA DE ESPÉCIES DE} ARTOCARPUS

Foram analisados 183 trabalhos com plantas do gênero Artocarpus, publicados na literatura especializada entre 1907 e 2012, descrevendo o isolamento e a identificação de 369 substâncias distintas em 34 espécies, que serviram de base para estudo. Atividades biológicas avaliadas para 25 espécies estãolistadas nas Tabelas 1 e 2 , destacandose: antioxidante; anti-inflamatória; antimalária; 

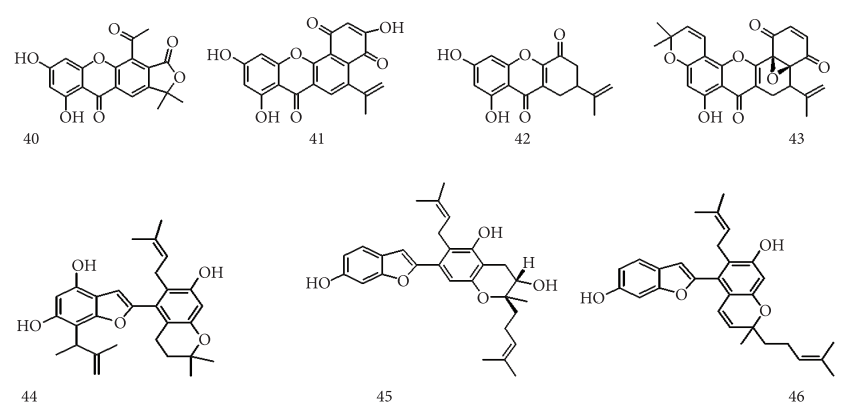

45
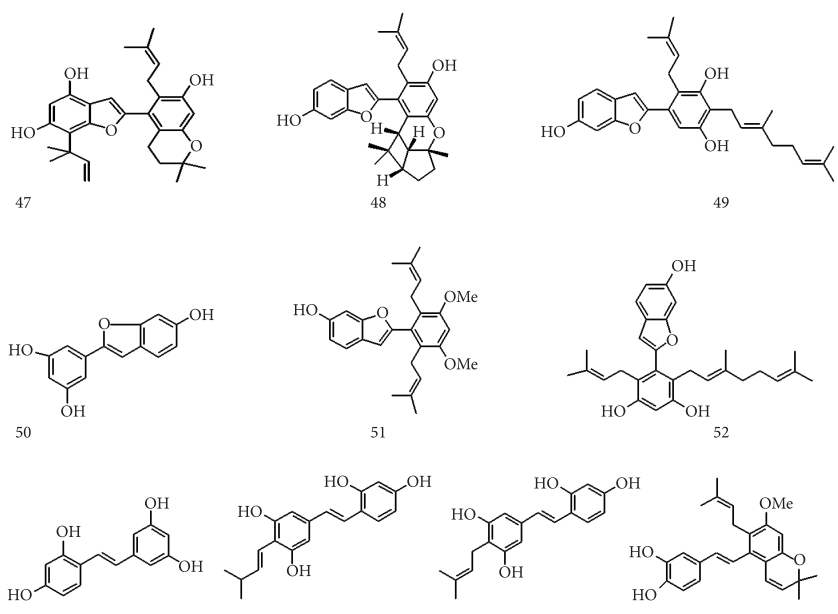

53
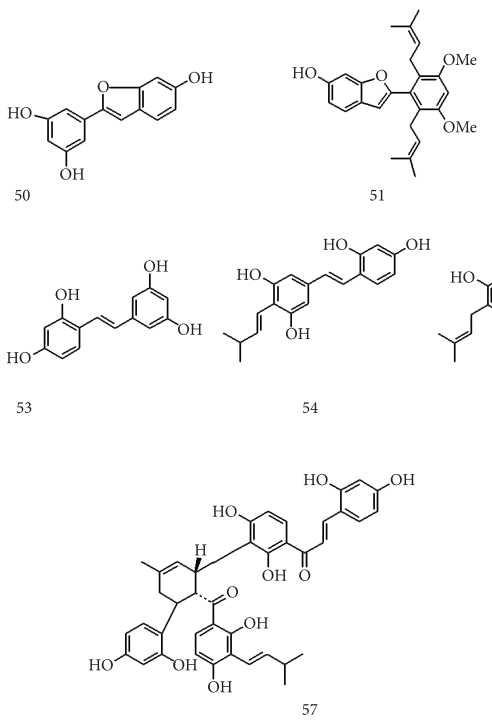

55

56

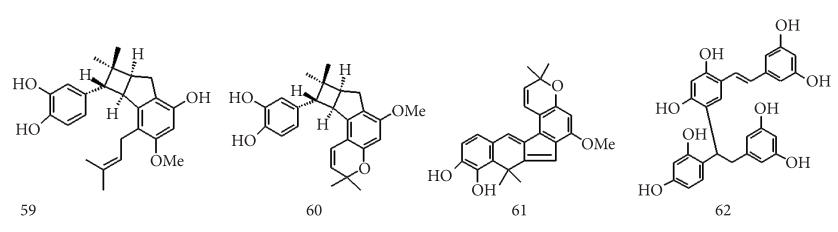

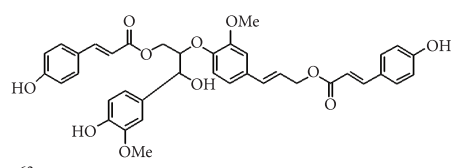

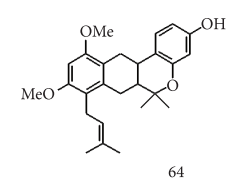

63

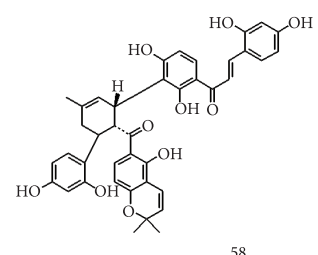
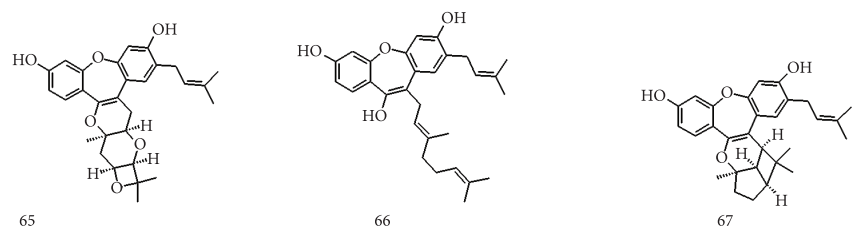

Figura 5. Estruturas típicas de substâncias fenólicas isoladas de espécies de Artocarpus. Figure 5. Typical phenolic compounds of Artocarpus species. 


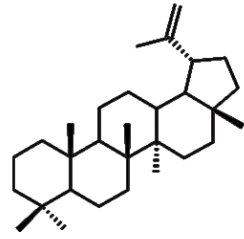

68

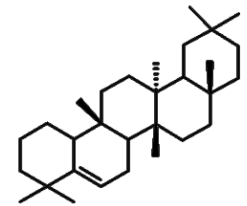

72

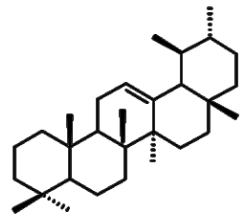

69

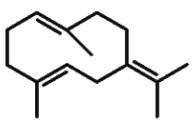

73

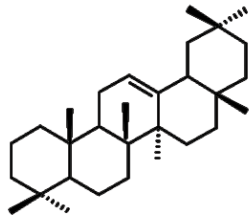

70

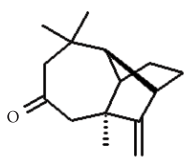

74

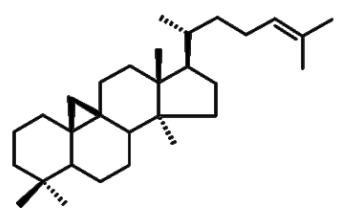

71

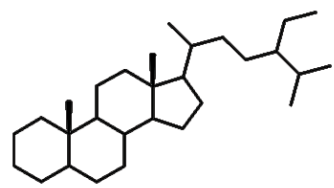

76

Figura 6. Esqueletos de terpenoides e fitoesteroides isolados de espécies de Artocarpus.

Figure 6. Terpenes and phytosteroids isolated of Artocarpus species.

citotóxica; anticancerígena; antiandrogênica; antiHIV; antileucêmica; despigmentante; antifúngica; antibacteriana; antiulcerogênica; anticoagulante; como marcadora de atividades tumorais em tecidos; imunossupressora; inibidora de catepsina, e antiherpética.

A osteoporose promove a diminuição da massa óssea, o que afeta a qualidade e a resistência dos ossos, culminando em fratura (Domiciano \& Pinheiro, 2011). Urge então encontrar alternativas para atenuar ou mesmo eliminar essa doença de caráter degenerativo. $\mathrm{O}$ extrato metanol/diclorometano (1:1) de brotos da espécie Artocarpus altilis mostrouse eficaz como agente antirreabsortivo ao inibir a catepsina K (CatK), uma cisteína-protease, peça fundamental na degradação das proteínas da matriz óssea (Patil et al., 2002). Há também estudos in vitro dessa espécie relacionando-a ao efeito citotóxico contra câncer e como citoprotetora das células mieloides U937 do sistema imune (Wang et al., 2007b, 2006).

A. chama apresentou também atividade antitumoral in vitro em linhagens de tumor de ovário $1 \mathrm{~A} 9$, de câncer de mama, MCF-7 e MDA-
MB-231, em células do cólon humano, HCT-8, e em melanoma humano, SK-MEL-2 (Wang et al., 2004). Algumas substâncias isoladas da espécie $A$. dadah foram eficazes na inibição das lesões causadas pelo 7,12-dimetilbenzo[b]fenantreno, DMBA, em uma cultura de células mamárias de ratos, o que torna o fato interessante para estudos posteriores na quimioprevenção do câncer (Su et al., 2002). Contra o carcinoma de pulmão humano em células A549, a espécie A. elasticus apresentou uma forte atividade citotóxica (Ko et al., 2005), enquanto que todos os flavonoides isolados de A. elasticus apresentaram atividade citotóxica de moderada a intensa contra três linhagens de câncer: MCF-7, TK-10 (em células renais) e em células epiteliais, UACC-62, um dos mais graves tipos de câncer de pele (Yamazaki et al., 1987). De A. kemando, foram isolados quatro flavonoides prenilados que apresentaram atividade citotóxica contra células tumorais de carcinoma nasofaringeal, KB (Seo et al., 2003), o que também foi verificado para um 2-arilbenzofurano de A. lakoocha isolado de suas raízes (Puntumchai et al., 2004) e para duas outras substâncias fenólicas de $A$. rigidus Blume subsp. rigidus ( $\mathrm{Lu}$ et al., 2007). Outros 2-arilbenzofuranos, 
Tabela 1. Classes das substâncias isoladas e atividade biológica do gênero Artocarpus.

Table 1. Artocarpus genus isolated compound classes and bioactivity.

\section{Espécies Substâncias/Extratos} estilbenos, 2-arilbenzofuranos

A. altilis triterpenos, esteroides, flavonoides geranilados

A. chama flavonoides prenilados

A. champeden flavonoides prenilados

A. communis flavonoides prenilados e geranilados

estilbenos prenilados, flavonoides, benzofuranos e neolignanas

\section{A. dadah}

A. elasticus

flavonoides prenilados

A. gomezianus

estilbenos, flavonoides,

2-arilbenzofuranos prenilados

A. heterophyllus flavonoides prenilados, polifenóis

e lecitinas

A. incisus

A. integer

flavonoides prenilados e geranilados

A. integra estilbeno prenilado

A. integrifolia catequinas

A. kemando

extrato, flavonoides prenilados

flavonoides prenilados e geranilados, e triterpenos

A. lakoocha

2-arilbenzofuranos, lecitinas e

flavonoides prenilados

A. lowii

flavonoides prenilados

flavonoides prenilados e

A. nobilis geranilados, xantonas, estilbenos prenilados e triterpenos

A. obtusus

xantonas

A. odoratissimus flavonoides e antocianinas

A. petelotii 2-arilbenzofuranos

A. rigidus flavonoides e xantonas

A. rotunda

flavonoides prenilados e geranilados

A. scortechini flavonoides prenilados

A. sepicanus flavonoides geranilados

A. teysmani flavonoides prenilados

A. tonkinensis flavonoides
Bioatividade

Material

Artigos

citotóxica e inibidora de catepsina

frutos, cascas do tronco, folhas e brotos

antitumoral raiz

5-6

antimalária, citotóxica e

tronco, cerne e raiz

antileucêmica

antioxidante, citotóxica,

antiagregante,

anti-inflamatória e

raiz, cerne folhas,

flores secas, casca do

antileucêmica tronco

anticâncer e anti-inflamatória caule e galhos

antileucêmica

antitumoral e citotóxica madeira e raiz

despigmentante, anti-HSV e raiz, cerne, folhas e anti-HIV

tronco

anti-inflamatória

hipoglicemiante,

despigmentante,

raiz, folhas frutos, antioxidante, anti HIV-I e alburno antiagregante

despigmentante $\mathrm{e}$ antiandrogênica

cerne e folhas

antimalária

partes aéreas 58

antiulcerogênica

folhas

antifúngica, antituberculose e sementes, cascas do hipoglicemiante tronco

antitumoral

caule

antibacteriana, antiagregante e anti- HIV

raiz, sementes e cerne $\quad 63-66$

antioxidante

folhas

$67-68$

antioxidante, antifúngica

e inibitória de

acetilcolinesterase

raiz, frutos, folhas e

caule

antioxidante, antimicrobiana

e inibidora de tirosinase tronco

antioxidante

frutos

74

antitumoral

tronco

75

antimicobacteriana e antitumoral

raiz

76

antileucêmica

raiz

77

antiagregante

folhas

78

antimicrobiana e

antifúngica

folhas

79

antiagregante

folhas

80

imunossupressora

folhas

81-82 
Tabela 2. Referências da Tabela 1.

Table 2. Table 1 references.

\begin{tabular}{|c|c|c|}
\hline Espécies & Artigos & Referências \\
\hline A. altilis & $1-4$ & Patil et al. (2002), Wang et al. $(2006,2007)$ e Boonphong et al. (2007) \\
\hline A. chama & $5-6$ & Wang et al. (2004) e Ahmed et al. (2012) \\
\hline A. champeden & $7-13$ & $\begin{array}{l}\text { Widyawaruyanti et al. (2007), Wahyuni et al. (2009), Syah et al. (2004, 2006), Hakim et al. } \\
\text { (1999), Achmad et al. (1996) e Kartini (2011) }\end{array}$ \\
\hline A. communis & $14-23$ & $\begin{array}{l}\text { Lotulung et al. (2008), Fang et al. (2008a), Lin et al. (2009, 2011), Weng et al., 2006, } \\
\text { Han et al. (2006), Wei et al. (2005), Koshihara et al. (1988), Hsu et al. (2011) e Kuete et al. } \\
\text { (2011) }\end{array}$ \\
\hline A. dadah & 24 & Su et al. (2002) \\
\hline A. elasticus & $25-28$ & Ko et al. (2005), Yamazaki et al. (1987), Musthapa et al. (2009) e Nascimento et al. (1997) \\
\hline A. gomezianus & $29-32$ & Likhitwitayawuid et al. $(2000,2001,2005 a, 2006)$ \\
\hline A. heterophyllus & $33-52$ & $\begin{array}{l}\text { Lako et al. (2007), Ko et al. (1998), Toda \& Shirataki (2006), Lin et al. (1996), Wei et al. } \\
\text { (2005), Arung et al. (2007, 2006), Fang et al. (2008b), Chandrika et al. (2006), Kabir } \\
\text { (1998), Jagtap et al. (2011), Loizzo et al., 2010, Lakheda et al. (2011), Patel \& Patel (2011), } \\
\text { Siritapetawee et al. (2012), Meshram et al. (2011), Omar et al. (2011), Gupta et al. (2011), } \\
\text { Shanmugapriya et al. (2011) e Navarro-García et al. (2012) }\end{array}$ \\
\hline A. incisus & $53-57$ & Shimizu et al. (2000a, b, c, 2002) e Pitaksuteepong et al. (2007) \\
\hline A. integer & 58 & Boonlaksiri et al. (2000) \\
\hline $\begin{array}{l}\text { A. integra } \\
\text { A. integrifolia }\end{array}$ & $\begin{array}{c}59 \\
60-61\end{array}$ & $\begin{array}{l}\text { Yamazaki et al. (1987) } \\
\text { Custodio et al. (2011) e Hema et al. (2011) }\end{array}$ \\
\hline A. kemando & 62 & Seo et al. (2003) \\
\hline A. lakoocha & $63-66$ & $\begin{array}{l}\text { Puntumchai et al. (2004), Likhitwitayawuid et al. (2005b), Chattekjw et al. (1988) e } \\
\text { Suwannalert et al. (2012) }\end{array}$ \\
\hline A. lowii & $67-68$ & Jamil et al. (2008) e Jantan et al. (2010) \\
\hline $\begin{array}{l}\text { A. nobilis } \\
\text { A. obtusus }\end{array}$ & $\begin{array}{c}69-72 \\
73\end{array}$ & $\begin{array}{l}\text { Jayasinghe et al. (2004a, b, 2006, 2008) } \\
\text { Hashim et al. (2012) }\end{array}$ \\
\hline A. odoratissimus & 74 & Bakar et al. (2009) \\
\hline A. petelotii & 75 & Hong \& Ai-Jun (2008) \\
\hline A. rigidus & 76 & Lu et al. (2007) \\
\hline $\begin{array}{l}\text { A. rotunda } \\
\text { A. scortechini }\end{array}$ & $\begin{array}{l}77 \\
78\end{array}$ & $\begin{array}{l}\text { Suhartati et al. (2001) } \\
\text { Jantan et al. (2010) }\end{array}$ \\
\hline $\begin{array}{l}\text { A. sepicanus } \\
\text { A. teysmani }\end{array}$ & $\begin{array}{l}79 \\
80\end{array}$ & $\begin{array}{l}\text { Radwan et al. (2009) } \\
\text { Jantan et al. (2010) }\end{array}$ \\
\hline A. tonkinensis & $81-82$ & Thuy et al. (2004) e Dang et al., (2009) \\
\hline
\end{tabular}

também isolados de raízes derivadas de A. petelotii, apresentaram atividade antitumoral em linhagens de células hepáticas HepG e de mama, MCF-7 (Hong \& Ai-Jun, 2008). A. rigidus Blume subsp. rigidus também se mostrou ativo contra duas outras linhagens de câncer, sendo uma de mama e outra de pulmão, NCI-H187 (Lu et al., 2007).

Testes in vitro e in vivo (Widyawaruyanti et al., 2007; Wahyuni et al., 2009) demostraram que os flavonoides prenilados de A. champeden Spreng. apresentaram significativa inibição dos parasitas Plasmodium falciparum e $P$. berghei, assim como os três estilbenos isolados de Artocarpus interger sobre o primeiro parasita (Boonlaksiri et al., 2000), ssendo então uma opção promissora na luta contra a malária, uma doença infecciosa aguda que atinge 300 milhões de pessoas por ano, com um número de mortes em torno de 1 a 1,5 milhão (Gurgel et al., 2007). As espécies $A$. champeden, A. elasticus, A. communis e $A$. rotunda apresentaram ainda uma forte atividade citotóxica contra células P-388 provenientes de leucemia murina, em testes realizados in vitro (Syah et al., 2004, 2006; Hakim et al., 1999; Musthapa et al., 2009; Lotulung et al., 2008; Suhartati et al., 2001). A toxidez contra o crustáceo Artemia salina (Achmad et al., 1996) corroborou com a presença de substâncias bioativas com atividade antitumoral de A. champeden (McLaughlin et al., 
1998). Das folhas de A. communis, foram isoladas cinco chalconas geraniladas, que induziram in vitro a apoptose em células SW872 responsáveis pelo lipossarcoma humano, sendo a substância isolespeol a de maior potencial (Fang et al., 2008a).

Há também um registro de que as substâncias isoladas de $A$. communis possuem efeito protetor contra os danos oxidativos do DNA in vitro, o que indica serem essas substâncias antioxidantes; esta propriedade é verificada também para as espécies $A$. heterophyllus, A. lowii, A. nobilis e A. odorantissimus (Lin et al., 2009; Lako et al., 2007; Ko et al., 1998; Toda \& Shirataki, 2006; Jamil et al., 2008; Jayasinghe et al., 2004a, b, 2006, 2008; Bakar et al., 2009; Hashim et al., 2012; Gupta et al., 2011; Omar et al., 2011; Jagtap et al., 2011; Loizzo et al., 2010; Meshram et al., 2011; Suwannalert et al., 2012).

Das raízes de $A$. communis, foram isoladas três flavonas preniladas que inibiram a agregação plaquetária impedindo a formação de tromboxanos, característica essa comum também para $A$. heterophyllus. Essa atividade torna essas espécies interessantes para o controle de trombose e embolia (Weng et al., 2006; Lin et al., 1996). Em estudos in vivo, substâncias isoladas de $A$. communis e $A$. heterophyllus inibiram a produção de óxido nítrico pelas células RAW 264-7 e por macrófagos ativados com lipopolissacarídeos; ou seja, essas espécies mostram-se bastante promissoras para estudos mais minunciosos, que poderiam relacioná-las à diminuição de processos inflamatórios, isquemia e vasodilatação (Han et al., 2006; Wei et al., 2005). Outros processos inflamatórios foram estudados in vivo e in vitro com substâncias isoladas de $A$. communis, $A$. heterophyllus e $A$. dadah, sendo estas mais uma alternativa para o alívio de dores (Su et al., 2002; Wei et al., 2005; Koshihara et al., 1988; Kakheda et al., 2011).

Arcarpus gomezianus Wall. e A. incisus Lin. (Shimizu et al., 2000a) apresentaram atividade inibidora de tirosinase, uma importante enzima na biossíntese do pigmento melanina (Likhitwitayawuid et al., 2000, 2001), sendo que a primeira espécie apresentou ainda ação anti-herpes dos tipos 1 e 2, e anti-HIV (Likhitwitayawuid et al., 2005a, 2006). Com pouca ou nenhuma toxidez, estudos in vivo de A. heterophyllus mostraram-se eficazes na inibição da biossíntese de melanina em células B16 de melanoma, sendo os flavonoides prenilados ou hidroxilados em posições específicas, os responsáveis por essa bioatividade (Arung et al., 2006, 2007). O extrato metanólico do cerne de A. incisus também apresentou várias substâncias com potencial de inibir a tirosinase e, em especial, uma flavona denominada artocarpina, [6-(3-metil-1-butenil)-5,2',4'-triidróxi-3-isoprenil7-metóxiflavona], que, apesar de não possuir essa propriedade, foi efetiva no clareamento da pele de roedores hiperpigmentada por efeito da radiação UVB (Shimizu et al., 2002).

A flavona artocarpesina, 5,7,2',4'-tetrahidróxi6-(3-metilbut-3-enil), presente nas espécies $A$. heterophyllus (Fang et al., 2008b), A. incisus e A. elasticus, em função dos registros apresentados, parece ser uma boa opção às desordens de origem inflamatória (Yamazaki et al., 1987; Shimizu et al., 2000a, b; Nascimento et al., 1997).

A fração etanólica solúvel em acetato de etila contendo $2 \mathrm{~g}$ de flavonoides em $200 \mathrm{~g}$ de folhas frescas de $A$. heterophyllus apresentou poderosa ação antiglicêmica in vivo, quando comparada ao uso de tolbutamida, um hipoglicemiante oral da família das sulfonilureias muito utilizado em tratamentos do Diabetis mellitus tipo II (Chandrika et al., 2006). Em uma estimativa recente (Hocayen, 2012), cerca de 257 milhões de pessoas foram acometidas dessa doença pelo mundo, sendo dez milhões só no Brasil; este valor tende a aumentar, principalmente nos países em desenvolvimento nos próximos anos. Na medida em que a presença dessa árvore é grande nas regiões tropicais do planeta, sua utilização como fitoterápico parece ter grande perspectiva, necessitando, porém, de mais estudos que confirmem sua atuação como tal.

Mais de $50 \%$ do teor proteico das sementes da jaqueira é formado por uma glicoproteína denominada jacalina, que, por ser mitogênica apenas para células $\mathrm{CD} 4+$ presentes na membrana celular, tem papel importante no estudo do HIV-I (Kabir, 1998). Com teor elevado dessa lecitina, associada à outra, extratos das sementes de Artocarpus integrifolia apresentaram-se efetivos in vivo na resposta imunológica contra Candida albicans (Custodio, 2011). 
Substâncias encontradas em $A$. incisus inibiram a enzima 5-alpha-redutase, o que seria um indício que poderiam ser utilizadas em desordens androgênicas, como acne, alopecia, seborreia e hirsutismo (Shimizu et al., 2000a; Pitaksuteepong et al., 2007).

Catequinas isoladas de folhas de $A$. integra apresentaram atividade ulcerogênica (Yamazaki et al., 1987) e, das raízes de A. lakoocha, duas substâncias da classe dos 2- arilbenzofuranos apresentaram in vivo atividade antimicobacteriana (Puntumchai et al., 2004), atividade essa também presente em A. rigidus Blume subsp. Rigidus, que se mostrou eficaz contra o Mycobacterium tuberculosis, causador da tuberculose, uma doença infecciosa (Namdaung et al., 2006). Os extratos metanólicos e butanólicos de A. nobilis de folhas e de cascas do caule, respectivamente, apresentaram atividade contra Cladosporium cladosporioides, um fungo entomopatogênico de ocorrência natural no Reino Vegetal (Oliveira et al., 2004). Triterpenos de esqueleto cicloartano, dessa mesma espécie, também apresentaram uma inibição de acetilcolinesterase (Jayasinghe et al., 2004b; Hano et al., 1990).

O estilbeno oxiresveratrol, substância majoritária do cerne de A. lakoocha, apresentou moderada ação anti HIV-1, em estudo que o compara ao efeito surtido pela droga azidotimidina, AZT (Likhitwitayawuid et al., 2005a). Auronas glicosiladas isoladas da espécie A. tonkinensis apresentaram atividade imunossupressora, o que é perfeitamente compatível com o uso popular de seu extrato de folhas cruas no Vietnam para o tratamento de doenças autoimunes, como a artrite reumatoide e o lúpus eritematoso, entre outras (Thuy et al., 2004).

As espécies $A$. venenosa, A. rigida, A. bracteata, A. fretessi, A. hirsutus, A. hypargyreus, A. incisa, A. thailandicus e $A$. lanceifolius ainda não foram analisadas quanto a possíveis atividades biológicas.

\section{CONCLUSÕES}

As espécies do gênero Artocarpus são bastante ricas em substâncias fenólicas, que para as plantas podem servir para amenizar o estresse oxidativo a que são submetidas ou ainda como defesa contra herbívoros ou agentes patogênicos. Esses representantes vegetais apresentam-se ao homem, generosamente, como mais uma opção para estudos de natureza farmacológica, visando a encontrar soluções principalmente para as doenças degenerativas, como o câncer e o diabetes, entre várias outras.

Tendo em vista o uso dessas plantas na medicina popular de vários países asiáticos, seriam interessantes análises fitoquímicas e farmacológicas envolvendo um maior número de espécies do gênero Artocarpus para a confirmação de seu potencial biológico e sua posterior utilização, pois $68 \%$ das espécies foram estudadas e, destas, cerca de $74 \%$ apresentaram bioatividade in vivo ou in vitro, até o momento da presente pesquisa.

Há várias recomendações e trabalhos publicados baseados no controle de espécies exóticas, elaborados pelo Programa Global de Espécies Invasoras - Global Invasive Species Programme, GISP -, que vão desde o manejo assistido até a total erradicação (Machado et al., 2009; Oliveira \& Pereira, 2010). Poder-se-ia ainda minimizar o impacto causado ao meio ambiente por uma espécie invasora - como é, principalmente, no caso do Brasil, o da espécie Artocarpus heterophyllus - utilizando-a para estudos criteriosos, pois há inúmeros artigos relacionando-a a atividades anti-inflamatória, antiglicêmica, despigmentante, antioxidante, antiHIV-I e antiagregante. Se, por um lado, essa espécie oferece risco à flora nativa pelo seu caráter exótico e, por isso, há de ser controlada, por outro lado, esta poderia fornecer um vasto número de fitoterápicos para outros males que aflingem a sociedade. Ressalte-se que nem tudo o que parece ruim por uma perspectiva o será em outra; assim, partindo desse dualismo, juntamente com uma espécie exótica invasora, existe um potencial bioativo promissor para o desenvolvimento de novos fármacos, o que a torna, portanto, de grande valia.

\section{AGRADECIMENTOS}

UFRJ/ CNPq.

\section{STATUS DA SUBMISSÃO}

Recebido: 02/09/2012

Aceito: 07/01/2013

Publicado: 28/02/2013 


\section{AUTOR(ES) PARA CORRESPONDÊNCIA}

\section{Valéria de Jesus Pereira}

Núcleo de Pesquisas de Produtos Naturais, Universidade Federal do Rio de Janeiro - UFRJ, CEP 21941-902, Rio de Janeiro, RJ, Brasil e-mail: val.jp@ig.com.br

\section{REFERENNCIAS}

Achmad SA, Hakim EH, Juliawaty LD, Makmur L, Aimi SN, Ghisalberti EL. A new prenylated flavone from Artocarpus champeden. Journal of Natural Products 1996; 59(9): 878-879. http://dx.doi. org/10.1021/np960330q

Ahmed T, Uddin MN, Ahmed SF, Saha A, Farhana K, Rana MS. In vitro evaluation of antioxidant potential of Artocarpus chama Buch. Fruits. Journal of Applied Pharmaceutical Science 2012; 2(10): 75-80.

Arung ET, Shimizu K, Kondo R. Inhibitory effect of isoprenoid-substituted flavonoids isolated from Artocarpus heterophyllus on melanin biosynthesis. Planta Medica 2006; 72: 847-850. PMid:16732541. http://dx.doi.org/10.1055/s-2006-931606

Arung ET, Shimizu K, Kondo R. Structure-activity relationship of prenyl-substituted polyphenols from Artocarpus heterophyllus as inhibitors of melanin biosynthesis in cultured melanoma cells. Chemistry \& Biodiversity 2007; 4(9): 2166-2171. PMid:17886834. http://dx.doi.org/10.1002/cbdv.200790173

Bakar MFA, Mohamed M, Rahmat A, Fry J. Phytochemicals and antioxidant activity of different parts of bambangan (Mangifera pajang) and tarap (Artocarpus odoratissimus). Food Chemistry 2009; 113(2): 479-483. http://dx.doi.org/10.1016/j. foodchem.2008.07.081

Boonlaksiri C, Oonanant W, Kongsaeree P, Kittakoop P, Tanticharoen M, Thebtaranonth Y. An antimalarial stilbene from Artocarpus integer. Phytochemistry 2000; 54(4): 415-417. http://dx.doi. org/10.1016/S0031-9422(00)00074-1

Boonphong S, Baramee A, Kittakoop P, Puangsombat P. Antitubercular and antiplasmodial prenylated flavones from the roots of Artocarpus altilis. Chiang Mai Journal Science 2007; 34(3): 339-344.

Bremer B, Bremer K, Chase MW, Fay MF, Reveal JL, Soltis DE et al. An update of the angiosperm phylogeny group classification for the orders and families of flowering plants: APG III. Botanical Journal of the Linnean Society 2009; 161(2): 105-121. http://dx.doi. org/10.1111/j.1095-8339.2009.00996.x

Chandrika UG, Wedage WS, Wickramasinghe SMDN, Fernando WS. Hypoglycemic action of the flavonoid fraction of Artocarpus heterophyllus leaf. The African Journal of Traditional, Complementary and Alternative Medicines 2006; 3(2): 42-50. http://dx.doi.org/10.4314/ ajtcam.v3i2.31156

Chattekjw BP, Ahmed H, Chowdhury S. Further characterization of Artocarpus lakoocha lectin Artocarpin purified using rivanol. Carbohydrate Research 1988; 180: 97-110. http://dx.doi. org/10.1016/0008-6215(88)80067-3

Custodio LA, Loyola W, Conchon-Costa I, Quirino GFS, Felipe I. Protective effect of Artin M from extract of Artocarpus integrifolia seeds by Th1 and Th17 immune response on the course of infection by Candida albicans. International Immunopharmacology 2011; 11(10): 1510-1515. PMid:21609786. http://dx.doi.org/10.1016/j. intimp.2011.05.005

Dang DTN, Eriste E, Liepinsh E, Trinh TT, ErlandssonHarris H, Sillard R et al. A novel antiinflammatory compound, antonkin-4'-O-glucoside, from the leaves of Artocarpus tonkinensis suppresses experimentally induced arthritis. Scandinavian Journal of Immunology 2009; 69: 110-118. PMid:19170963. http:// dx.doi.org/10.1111/j.1365-3083.2008.02205.x

Datwyler SL, Weiblen GD. On the origin of the fig: phylogenetic relationships of Moraceae from ndhF sequences. American Journal of Botany 2004; 91: 767-777. PMid:21653431. http:// dx.doi.org/10.3732/ajb.91.5.767

Domiciano DS, Pinheiro MM. A prática clínica em doenças reumáticas - Osteoporose. Revista Brasileira de Medicina 2011; 68(5): 141-149.

Fang S-C, Hsu C-L, Yu Y-S, Yen G-C. Cytotoxic effects of new geranyl chalcone derivatives isolated from the leaves of Artocarpus communis in SW 872 human liposarcoma cells. Journal of Agricultural and Food Chemistry 2008a; 56(19): 8859-8868. PMid:18767861. http://dx.doi.org/10.1021/jf8017436

Fang S-C, Hsu C-L, Yen G-C. Anti-inflammatory effects of phenolic compounds isolated from the fruits of Artocarpus heterophyllus. Journal of Agricultural and Food Chemistry 2008b; 56(12): 4463-4468. PMid:18500810. http://dx.doi.org/10.1021/jf800444g

Gupta D, Mann S, Sood A, Gupta RK. Phytochemical, nutritional and antioxidant activity evaluation of seeds of jackfruit (Artocarpus heterophyllus Lam.). International Journal of Pharma and Bio Sciences 2011; 2(4): 336-345.

Gurgel H, Zaninetti J-M, Fotsing J-M. Estudo de casos de malária em Roraima a partir de técnicas de análise especial. In: Anais do XIII Simpósio Brasileiro de Sensoriamento Remoto; 2007; Florianópolis. INPE; 2007.

Hakim EH, Fahriyati A, Kau MS, Achmad SA, Makmur L, Ghisalberti E et al. Artoindonesianins $\mathrm{A}$ and $\mathrm{B}$, two new prenylated flavones from the 
root of Artocarpus champeden. Journal of Natural Products 1999; 62(4): 613-615. PMid:10217722. http:// dx.doi.org/10.1021/np9802791

Han A-R, Kang Y-J, Windono T, Lee S-H, Seo E-K. Prenylated flavonoids from the heartwood of Artocarpus communis with inhibitory activity on lipopolysaccharide-induced nitric oxide production. Journal of Natural Products 2006; 69(4): 719-721. PMid:16643064. http://dx.doi.org/10.1021/np0600346

Hano Y, Yamagami Y, Kobayashi M, Isohata R, Nomura T. Artonins E and F, two new prenylflavones from the bark of Artocarpus communis Forst. Heterocycles 1990; 31(5): 877-882. http://dx.doi. org/10.3987/COM-90-5350

Hashim NM, Rahmani M, Cheng LG, Sukari MA, Yahayu M, Amin MAM et al. Antioxidant, antimicrobial and tyrosinase inhibitory activities of xanthones isolated from Artocarpus obtusus F.M. Jarrett. Molecules 2012; 17: 6071-6082. PMid:22614861. http:// dx.doi.org/10.3390/molecules 17056071

Hema MR, Ramaiah M, Vaidya VP, Shivakumar BS, Begum A. Evaluation of antidiabetic and antitubercular activities of methanol extract of root bark of Artocarpus integrifolia. Research Journal of Pharmaceutical, Biological and Chemical Sciences 2011; 2(4): 886-893.

Hocayen PAS. Efeito da administração oral do extrato de Baccharis dracunculifolia na obesidade induzida por glutamato monossódico (MSG) [dissertação]. Ponta Grossa: Universidade Estadual de Ponta Grossa, Universidade Estadual do Centro Oeste; 2012.

Hong S, Ai-Jun H. Prenylated 2-arylbenzofurans from Artocarpus petelotii. Natural Products Research 2008; 22(16): 1451-1456. PMid:19023808. http://dx.doi.org/10.1080/14786410802076374

Hsu C-L, Shyu M-H, Lin J-A, Yen, G-C, Fang S-C. Cytotoxic effects of geranyl flavonoid derivatives from the fruit of Artocarpus communis in SKHep-1 humana hepatocellular carcinoma cells. Food Chemistry 2011; 127(1):127-134. http://dx.doi. org/10.1016/j.foodchem.2010.12.100

Instituto Hórus de Desenvolvimento e Conservação Ambiental. Base de dados nacional de espécies exóticas invasoras. Instituto Hórus de Desenvolvimento e Conservação Ambiental, Florianópolis; 2012. Available from: http://i3n.institutohorus.org.br.

Jagtap UB, Wagmare SR, Lokhande VH, Supprasanna $\mathrm{P}$, Bapat VA. Preparation and evaluation of antioxidant capacity of jackfruit (Artocarpus heterophyllus Lam.) wine and its protective role against radiation induced DNA damage. Industrial Crops and Products 2011; 34(3). http://dx.doi.org/10.1016/j.indcrop.2011.05.025

Jantan I, Yasin YHM, Jamil S, Sirat H, Basar N. Effect of prenylated flavonoids and chalcones isolated from Artocarpus species on platelet aggregation in human whole blood. Journal of Natural Medicines 2010; 64(3): 385-369. PMid:20349149. http:// dx.doi.org/10.1007/s11418-010-0410-0

Jamil S, Sirat HM, Jantan I, Aimi N, Kitajima M. A new prenylated dihydrochalcone from the leaves of Artocarpus lowii. Journal of Natural Medicines 2008; 62(3): 321-324. PMid:18404311. http:// dx.doi.org/10.1007/s11418-008-0226-3

Jayasinghe L, Balasooriya BAIS, Padmini WC, Hara N, Fujimoto Y. Geranyl chalcone derivatives with antifungal and radical scavenging properties from the leaves of Artocarpus nobilis. Phytochemistry 2004a; 65(9): 1287-1290. PMid:15184014.http://dx.doi.org/10.1016/j. phytochem.2004.03.033

Jayasinghe L, Rupasinghe GK, Hara N, Fujimoto Y. P Geranylated phenolic constituents from the fruits of Artocarpus nobilis. Phytochemistry 2006; 67(13): 1353-1358. PMid:16730759. http://dx.doi. org/10.1016/j.phytochem.2006.04.011

Jayasinghe ULB, Puvanendrana S, Harab N, FujimotoY. Stilbene derivatives with antifungal and radical scavenging properties from the stem bark of Artocarpus nobilis. Natural Products Research 2004b; 18(6): 571-574. PMid:15595616. http://dx.doi.org/10.1080/1478641031 0001643867

Jayasinghe ULB, Samarakoon TB, Kumarihamy BMM, Hara N, Fujimoto Y. Four new prenylated flavonoids and xanthones from the root bark of Artocarpus nobilis. Fitoterapia 2008; 79(1): 37-41. PMid:17855020. http:// dx.doi.org/10.1016/j.fitote.2007.07.014

Kabir S. Jacalin: a jackfruit (Artocarpus heterophyllus) seed-derived lectin of versatile applications in immunobiological research. Journal of Immunological Methods 1998; 212: 193-211. http://dx.doi.org/10.1016/ S0022-1759(98)00021-0

Kartini, P. Immunomodulator activity of an isolate from Artocarpus champeden Spreng. on cytotoxicity function of $\mathrm{Cd} 8+$ of mice. In: International Seminar on Translational Research in Cancer Chemoprevention; 2011; Serpong. Indonesia; October 2011. p. 11-12.

Ko FN, Cheng ZJ, Lin CN, Teng CM. Scavenger and antioxidant properties of prenylflavones isolated from Artocarpus heterophyllus. Free Radical Biology and Medicine 1998; 25(2): 160-168. http://dx.doi. org/10.1016/S0891-5849(98)00031-8

Ko H-H, Lu Y-H, Yang S-Z, Won S-J, Lin C-N. Cytotoxic prenylflavonoids from Artocarpus elasticus. Journal of Natural Products 2005; 68(11): 1692-1695. PMid:16309328. http://dx.doi.org/10.1021/np050287j

Koshihara Y, Fujimoto Y, Inoue H. A new 5-lipoxygenase selective inhibitor derived from Artocarpus communis strongly inhibits arachidonic acid-induced ear edema. Biochemical Pharmacology 1988; 37: 2161-2165. http:// dx.doi.org/10.1016/0006-2952(88)90576-X 
Kuete V, Ango PY, Fotso GW, Kapche GDWF, Dzoyem JP, Wouking AG et al. Antimicrobial activities of the methanol extract and compounds from Artocarpus communis (Moraceae). Complementary and Alternative Medicine 2011; 11:42. PMid:21612612 PMCid:3118951. http://dx.doi.org/10.1186/1472-6882-11-42

Lakheda S, Devalia R, Jain UK, Gupta N, Raghuwansi AS, Patidar N. Anti-inflammatory activity of Artocarpus heterophyllus bark. Der Pharmacia Sinica 2011; 2(2): 127-130.

Lako J, Trenerry VC, Wahlqvist M, Wattanapenpaiboon N, Sotheeswaran S, Premier R. Phytochemical flavonols, carotenoids and the antioxidant properties of a wide selection of Fijian fruit, vegetables and other readily available foods. Food Chemistry 2007; 101(4): 1727-1741. http://dx.doi. org/10.1016/j.foodchem.2006.01.031

Likhitwitayawuid K, Sritularak B, De-Eknamkul W. Tyrosinase inhibitors from Artocarpus gomezianus. Planta Medica 2000; 66: 275-277. PMid:10821057. http://dx.doi.org/10.1055/s-2000-8656

Likhitwitayawuid K, Sritularak B. A new dimeric stilbene with tyrosinase inhibitory activity from Artocarpus gomezianus. Journal of Natural Products 2001; 64: 1457-1459. PMid:11720533. http:// dx.doi.org/10.1021/np0101806

Likhitwitayawuid K, Supudompol B, Sritularak B, Lipipun V, Rapp K, Schinazi, RF. Phenolics with anti-HSV and anti-HIV activities from Artocarpus gomezianus, Mallotus pallidus, and Triphasia trifolia. Pharmaceutical Biology 2005a; 43(8): 651-657. http:// dx.doi.org/10.1080/13880200500383058

Likhitwitayawuid K, Sritularak B, Benchanak K, Lipipun V, Mathew J, Raymond F. Phenolics with antiviral activity from Millettia erythrocalyx and Artocarpus lakoocha. Natural Product Research 2005b; 19(2): 177-182. PMid:15715263. http://dx.doi.org/10.1080/1478641041 0001704813

Likhitwitayawuid K, Saowalak Chaiwiriyaa S, Sritularaka B. Antiherpetic flavones from the heartwood of Artocarpus gomezianus. Chemistry \& Biodiversity 2006; 3: 1138-1143. PMid:17193228. http:// dx.doi.org/10.1002/cbdv.200690115

Lin K-W, Liu C-H, Tu H-Y, Ko H-H, Wei B-L. Antioxidant prenylflavonoids from Artocarpus communis and Artocarpus elasticus. Food Chemistry 2009; 115(2): 558-562. http://dx.doi. org/10.1016/j.foodchem.2008.12.059

Lin C-N, Lu C-M, Lin H-C, Fang S-C, Shieh B-J, Hsu M-F et al. Novel antiplatelet constituents from Formosan Moraceous plants. Journal of Natural Products 1996; 59(9): 834-838. PMid:8864236. http:// dx.doi.org/10.1021/np960376j
Lin JA, Fang SC, Wu CH, Huang SM, Yen GC. Anti-inflamatory effect of the 5,7,41-trihydroxy-6geranylflavanone isolated from the fruit of Artocarpus communis in S100B-induced human monocytes. Journal of Agricultural and Food Science 2011; 59(1): 105-111. PMid:21126004. http://dx.doi.org/10.1021/jf103455g

Loizzo MR, Tundis R, Chandrika UG. Antioxidant and antibacterial on foodborne pathogens of Artocarpus heterophyllus Lam. (Moraceae) leaves extracts. Journal of Food Science, 2010; 75(5): 291-295.

Lotulung PDN, Fajriah S, Hanafi M, Filaila E. Identification of cytotoxic compound from Artocarpus communis leaves against P-388 cells. Pakistan Journal of Biological Sciences 2008; 11(21): 2517-2520. PMid:19205275. http://dx.doi.org/10.3923/ pjbs.2008.2517.2520

Lu Y, Sun C, Wang Y, Pan Y. Two-dimensional counter-current chromatography for the preparative separation of prenylflavonoids from Artocarpus altilis. Journal of Chromatography A 2007; 1151(1-2): 31-36. PMid:17376457. http://dx.doi.org/10.1016/j. chroma.2007.02.099

Machado CJS, Oliveira AES, Matos DMS, Pivello V, Chame M, Souza RCCL et al. Recomendações para elaboração e consolidação de uma estratégia nacional de prevenção e controle das espécies exóticas no Brasil. Ciência e Cultura 2009; 61(1): 42-45.

McLaughlin JL, Rogers LL, Anderson JE. The use of biological assays to evaluate botanicals. Drug Information Journal 1998; 32(2): 513-524. http://dx.doi. org/10.1177/009286159803200223

Meshram R L, Umbarkar SN. Comparative evaluation for in vitro antioxidant activity from Artocarpus heterophyllus Lamk and Balanites aegyptiaca L. International Journal of PharmTech Research 2011; 3(4): 2006-2010.

Musthapa I, Juliawaty LD, Syah YM, Hakim EH, Latip J, Ghisalberti EL. An oxepinoflavone from Artocarpus elasticus with cytotoxic activity against P-388 cells Archives of Pharmacal Research 2009; 32(2): 191-194. PMid:19280147. http://dx.doi.org/10.1007/s12272-0091134-0

Namdaung U, Aroonrerk N, Suksamrarn S, Danwisetkanjana K, Saenboonrueng J, Arjchomphu $\mathrm{W}$ et al. Bioactive constituents of the root bark of Artocarpus rigidus subsp. rigidus. Chemical and Pharmaceutical Bulletin 2006; 54(10): 1433-1436. http:// dx.doi.org/10.1248/cpb.54.1433

Nascimento MSJ, Cidade H, Pinto M, Kijjoa A. Anticomplementary activity of prenylated flavones from Artocarpus elasticus. Pharmaceutical and Pharmacological Letters 1997; 7: 135-137.

Navarro-García VMl, Salinas-Sánchez DO, RodríguezFlores TA, Abarca-Vargas R, Aldana-Llanos L. 
Antimicrobial activity of artocarpesin from Artocarpus heterophyllus Lam. against methicillin-resistant Staphylococcus aureus (MRSA). Journal of Medicinal Plants Research 2012; 6(34): 4879-4882.

Nomura T, Hano Y, Aida M. Isoprenoid-substituted flavonoids from Artocarpus plants (Moraceae). Heterocycles 1998; 47: 1179-1205. http://dx.doi. org/10.3987/REV-97-SR(N)9

Oliveira AES, Pereira DG. Erradicação de espécies exóticas invasoras: múltiplas visões da realidade brasileira. Desenvolvimento e Meio Ambiente 2010; (21): 173-181.

Oliveira JS, Souza SE, Oliveira LL, Carvalho DA, Moreira DMO. Distribuição do fungo Cladosporium cladosporioides em regiões produtoras de café na Bahia. Bahia Agricola 2004; 6(3): 72-75.

Omar HS, El-Beshbishy HA, Moussa Z, Kamilia F, Taha KF, Singab ANB. Antioxidant activity of Artocarpus heterophyllus Lam. (Jack Fruit) leaf extracts: remarkable attenuations of hyperglycemia and hyperlipidemia in streptozotocin-diabetic rats. The Scientific World Journal 2011; 11: 788-800. PMid:21479350. http:// dx.doi.org/10.1100/tsw.2011.71

Patel RM, Patel SK. Cytotoxic activity of methanolic extract of Artocarpus heterophyllus against A549, Helaand MCF-7 cell lines. Journal of Applied Pharmaceutical Science, 2011; 1(7): 167-171.

Patil AD, Freyer AJ, Killmer L, Offen P, Taylor PB, Votta $\mathrm{BJ}$ et al. A new dimeric dihydrochalcone and a new prenylated flavone from the bud covers of Artocarpus altilis: potent inhibitors of cathepsin K. Journal of Natural Products 2002; 65(4): 624-627. PMid:11975520. http://dx.doi.org/10.1021/np0105634

Peixoto AM, Toledo FF. Enciclopédia agrícola brasileira. São Paulo: Edusp: FAPESP; 2002.

Pitaksuteepong T, Somsiri A, Waranuch N. Target transfolicular delivery of artocarpin extract of Artocarpus incisus by means of microparticles. European Journal of Pharmaceutics and Biopharmaceutics 2007; 67(3): 639-645. PMid:17493791. http://dx.doi.org/10.1016/j.ejpb.2007.03.019

Prestes MEB. A investigação da natureza no Brasil colônia. São Paulo: Annablume, Fapesp; 2000.

Puntumchai A, Kittakoop P, Rajviroongit S, Vimuttipong S, Likhitwitayawuid K, Thebtaranonth Y. Lakoochins $\mathrm{A}$ and $\mathrm{B}$, new antimycobacterial stilbene derivatives from Artocarpus lakoocha. Journal of Natural Products 2004; 67(3): 485- 486. PMid:15043440. http:// dx.doi.org/10.1021/np030429e

Radwan MM, Rodriguez-Guzman R, Manly SP, Jacob M, Ross SA. Sepicanin A - A new geranyl flavanone from Artocarpus sepicanus with activity against methicillin-resistant Staphylococcus aureus (MRSA).
Phytochemistry Letters 2009; 2(4): 141-143. http:// dx.doi.org/10.1016/j.phytol.2009.04.002

Santos AR, Bergallo HG, Rocha, CFD. Paisagem urbana alienígena. Ciência Hoje 2008; 41: 68-73.

Seo E-K, Lee D, Shin YG, Chai H-B, Navarro HA, Kardono LBS et al. Bioactive prenylated flavonoids from the stem bark of Artocarpus kemando. Archives of Pharmacal Research 2003; 26(2): 124-127. PMid:12643587. http://dx.doi.org/10.1007/BF02976656

Shanmugapriya K, Saravana PS, Payal H, Mohammed SP, Bennai W. A comparative study of antimicrobial potential and phytochemical analysis of Artocarpus heterophyllus and Manilkara zapota seed extracts. Journal of Pharmacy Research 2011; 4(8): 2587-2589.

Siqueira JC. Bioinvasão vegetal: Dispersão e propagação de espécies nativas e invasoras exóticas no campus da Pontifícia Universidade Católica do Rio de Janeiro (PUC-RJ). Pesquisas, Botânica 2006; 57: 319-330.

Siritapetawee S, Thammasirirak S, Samosornsuk W. Antimicrobial activity of a $48-\mathrm{kDa}$ protease (AMP48) from Artocarpus heterophyllus latex. European Review for Medical and Pharmacological Sciences 2012; 16: 132-137. Pmid:22338560.

Shimizu K, Fukuda M, Kondo R, Sakai K. The 5 alphareductase inhibitory components from heartwood of Artocarpus incisus: structure-activity investigations. Planta Medica 2000a; 66(1): 16-19. PMid:10705727. http://dx.doi.org/10.1055/s-2000-11114

Shimizu K, Kondo R, Sakai K, Buabarn S, Dilokkunanant U. A geranylated chalcone with 5-alphareductase inhibitory properties from Artocarpus incisus. Phytochemistry 2000b; 54(8): 737-739. http://dx.doi. org/10.1016/S0031-9422(00)00187-4

Shimizu K, Kondo R, Sakai K, Takeda N, Nagahata T. The skin-lightening effects of Artocarpin on UVB-induced pigmentation. Planta Medica 2002; 68(1): 79-81. PMid:11842337. http:// dx.doi.org/10.1055/s-2002-20057

Shimizu K, Yoshikawa K, Kondo K, Sakai K. The inhibitory components from Artocarpus incisus on melanin biosynthesis. Tennen Yuki Kagobutsu Toronkai Koen Yoshishu 2000c; 42: 535-539.

Su B-N, Cuendet M, Hawthorne ME, Kardono LBS, Riswan S, Fong HHS et al. Constituents of the bark and twigs of Artocarpus dadah with cyclooxygenase inhibitory activity. Journal of Natural Products 2002; 65(2): 163-169. PMid:11858749. http:// dx.doi.org/10.1021/np010451c

Suhartati T, Achmad SA, Aimi N, Hakima EH, Kitajima M, Takayama $\mathrm{H}$ et al. Artoindonesianin L, a new prenylated flavone with cytotoxic activity from Artocarpus rotunda. Fitoterapia 2001; 72(8): 912-918. http://dx.doi.org/10.1016/S0367-326X(01)00343-4 
Suwannalert P, Povichit N, Puchadapirom P, Junking M. Anti-aging activity and non-toxic dose of phytooxyresveratrol from Artocarpus lakoocha Roxb. Tropical Journal of Pharmaceutical Research 2012; 11 (1): 69-74. http://dx.doi.org/10.4314/tjpr.v11i1.9

Syah YM, Juliawaty LD, Achmad SA, Hakim EH, Ghisalberti EL. Cytotoxic prenylated flavones from Artocarpus champeden. Journal of Natural Medicines 2006; 60: 308-312. http://dx.doi.org/10.1007/ s11418-006-0012-Z

Syah YM, Achmada SA, Ghisalberti EL, Hakima EH, Mujahidin D. Two new cytotoxic isoprenylated flavones, artoindonesianins $\mathrm{U}$ and $\mathrm{V}$, from the heartwood of Artocarpus champeden. Fitoterapia 2004; 75(2): 134140. PMid:15030917. http://dx.doi.org/10.1016/j. fitote.2003.11.005

Thuy TT, Kamperdick C, Ninh PT, Lien TP, Thao TTP, Sung TV. Immunosuppressive auronol glycosides from Artocarpus tonkinensis. Pharmazie 2004; 59: 297-300. Pmid:15125577.

Toda S, Shirataki Y. Inhibitory effect of prenylated flavonoid in Euchresta japonica and Artocarpus heterophyllus on lipid peroxidation by interaction of hemoglobin and hydrogen peroxide. Pharmaceutical Biology (Philadelphia, PA, United States) 2006; 44: 271273.

Wahyuni TS, Ekasari W, Widyawaruyanti A, Hirasawa Y, Morita H, Zaini NC. Artopeden A, a new antiplasmodial isoprenylated flavone from Artocarpus champeden. Heterocycles 2009; 79(1): 1121-1126. http:// dx.doi.org/10.3987/COM-08-S(D)72

Wang H-W, Lu X, Liu Y. Analysis of volatile oil from the leaves of Artocarpus heterophyllus Lamk. By GC-MS. Shizhen Guoyi Guoyao 2007a; 18: 1596-1597.

Wang Y, Deng T, Lin L, Pan Y, Zheng X. Bioassay-guided isolation of antiatherosclerotic phytochemicals from Artocarpus altilis. Phytotherapy
Research 2006; 20(12): 1052-1055. PMid:17006973. http://dx.doi.org/10.1002/ptr.1990

Wang Y-H, Hou A-J, Chen L, Chen D-F, Sun H-D, Zhao Q-S et al. New isoprenylated flavones, Artochamins A-E, and cytotoxic principles from Artocarpus chama. Journal of Natural Products 2004; 67(5): 757- 761. PMid:15165133. http://dx.doi.org/10.1021/np030467y

Wang Y, Xu K, Lin L, Pan Y, Zheng X. Geranyl flavonoids from the leaves of Artocarpus altilis. Phytochemistry 2007b; 68(9):1300-1306. PMid:17363017. http://dx.doi. org/10.1016/j.phytochem.2007.01.009

Wei B-L, Weng JR, Chiu P-H, Hung C-F, Wang J-P, Lin C-N. Antiinflammatory flavonoids from Artocarpus heterophyllus and Artocarpus communis. Journal of Agricultural and Food Chemistry 2005; 53(10): 3867-3871. PMid:15884809. http://dx.doi.org/10.1021/jf047873n

Weng J-R, Chan S-C, Lu Y-H, Lin H-C, Ko H-H, Lin C-N. Antiplatelet prenylflavonoids from Artocarpus communis. Phytochemistry 2006; 67(8): 824829. PMid:16516939. http://dx.doi.org/10.1016/j. phytochem.2006.01.030

Widyawaruyanti A, Kalauni SK, Awale S, Nindatu M, Zaini NC, Syafruddin D et al. New prenylated flavones from Artocarpus champeden, and their antimalarial activity in vitro. Journal of Natural Medicine 2007; 61: 410-413. http://dx.doi.org/10.1007/ s11418-007-0153-8

Yamazaki M, Okuyama E, Matsudo T, Takamaru T. Principles of Indonesian herbal drugs having an antiulcerogenic activity. I. Isolation and identification of (+/-) catechin from Artocarpus integra Merr. Yakugaku Zasshi 1987; 107(11): 914-916. Pmid:3443909.

Yao S, Min Z-D. Two new chalcones from the leaves of Artocarpus heterophyllus. Chinese Journal of Natural Medicines 2005; 3(4): 219-223. 